\title{
Breast Radiotherapy (RT) Using Tangential Fields (TgF): A Prospective Evaluation of the Dose Distribution in the Sentinel Lymph Node (SLN) Area as Determined Intraoperatively by Clip Placement
}

\author{
Yazid Belkacemi, MD, PhD ${ }^{1}$, Veronique Bigorie, $\mathrm{MD}^{2}$, Qiong PAN, $\mathrm{MD}^{1}$, Ryan Bouaita, $\mathrm{MD}^{\mathbf{1}}$, Frederic Pigneur, \\ $\mathrm{MD}^{3}$, Emmanuel Itti, $\mathrm{MD}, \mathrm{PhD}^{4}$, Hakima Badaoui, $\mathrm{MD}^{5}$, Elias Assaf, $\mathrm{MD}^{6}$, Philippe Caillet, $\mathrm{MD}^{7}$, Elie Calitchi, \\ $\mathrm{MD}^{1}$, and Romain Bosc, $\mathrm{MD}^{2}$
}

${ }^{1}$ AP-HP, GH Henri Mondor. Service d'Oncologie-Radiothérapie et Centre Sein Henri Mondor. Université Paris-Est Créteil (UPEC), Créteil, France; ${ }^{2}$ APHP, GH Henri Mondor. Service de Chirurgie Plastique et Centre Sein Henri Mondor. Université Paris-Est Créteil (UPEC), Créteil, France; ${ }^{3}$ APHP, GH Henri Mondor. Service d'Imagerie Médicale et Centre Sein Henri Mondor, Créteil, France; ${ }^{4}$ APHP, GH Henri Mondor. Service de Médecine Nucléaire et Centre Sein Henri Mondor. Université Paris-Est Créteil (UPEC), Créteil, France; ${ }^{5}$ APHP, GH Henri Mondor. Service d'Anatomo-Pathologie et Centre Sein Henri Mondor, Créteil, France; ${ }^{6}$ APHP, GH Henri Mondor. Service d'Oncologie Médicale et Centre Sein Henri Mondor, Créteil, France; ${ }^{7}$ APHP, GH Henri Mondor. Unité d'Oncogériatrie, Créteil, France

\begin{abstract}
Background. Randomized trials have established that patients with limited involvement of sentinel lymph node (SLN) do not require axillary lymph node dissection (ALND). The similar outcome in patients with $\leq 2$ positive SLN with or without additional ALND is attributed, in part, to tangential fields (TgF) RT. We evaluated the dose distribution in the SLN biopsy area (SLNBa) as determined intraoperatively by clips placement for radiotherapy (RT) optimization.

Methods. This prospective study included 25 patients who had breast conservation. Titanium clips were used intraoperatively to mark the SLNBa. All patients had 3D-conformal RT using standard $(\mathrm{STgF})$ or high tangential fields $(\mathrm{HTgF})$. Axillary levels, SLNBa, and organs at risk were contoured on a CT
\end{abstract}

Yazid Belkacemi, Elie Calitchi, Romain Bosc belong to AROME Association of Radiotherapy and Oncology of the Mediterranean Area (www.aromecancer.org). Francilian Breast Intergroup (FBI) Paris France

(C) The Author(s) 2014. This article is published with open access at Springerlink.com

First Received: 7 May 2014;

Published Online: 6 August 2014

Y. Belkacemi, MD, PhD

e-mail: yazid.belkacemi@hmn.aphp.fr scan. Dose distribution and overlap between $\mathrm{TgF}$ and target volumes were analyzed.

Results. The average doses delivered to axilla levels I-III and SLNBa were 25, 5, 2, and $33 \mathrm{~Gy}$, respectively. The average dose delivered to SLNBa was higher using HTgF with better coverage of the axilla. Only 12 of 25 patients ( $48 \%$ ) had their SLNBa completely covered by the TgF. There was no impact of TgF size on ipsilateral lung dose. The mean heart dose delivered using $\mathrm{STgF}$ was lower than $\mathrm{HTgF}$.

Conclusions. In the era of SLNB, axilla and SNLBa RT technique has to be standardized to deliver adequate dose. We recommend the use of $\mathrm{HTgF}$ or direct axillary RT techniques (such as in AMAROS trial) in patients with metastases in SLN without ALND completion, when only TgF are expected to cure potential residual disease in the axilla.

An extensive literature, including seven randomized trials and B32 trial update at 10 years, has established that axillary lymph node dissection (ALND) is not required in patients with negative sentinel lymph node biopsy (SLNB) in which axillary recurrence is rare. ${ }^{1,2}$ SNLB represents the standard procedure for patients with early breast cancer (BC) and clinically node-negative (cN0). Thus, Saint-Gallen guidelines state that ALND should not be completed in $\mathrm{CN} 0$ patients with one to two macrometastatic (MAC) in the SLNs after breastconserving surgery (BCS) and tangential field (TgF) 
radiotherapy $(\mathrm{RT}){ }^{3}$ The American Society of Clinical Oncology (ASCO) updated guidelines concluded recently that women with one to two metastatic SLNs planning to undergo BCS with whole breast radiation therapy (WBRT) should not undergo ALND. ${ }^{4}$

Moreover, in the ACOSOG Z0011 trial, 6-year outcome after BCS plus WBRT was equivalent in SLNB and ALND patients with $\leq 2$ positive SLNs. This equivalence was attributed to the potential cure of axillary residual disease with systemic therapy and $\mathrm{TgF}$ RT. ${ }^{5}$ While radiation parameters and dose distribution in the axilla were not reported in the initial publication, Jagsi et al. tried recently to detail radiation treatments from the 605 available RT report forms. ${ }^{5}$ No clear conclusions could be drawn from the analyses on whether additional regional nodal RT was necessary or beneficial for these patients. ${ }^{6}$ The utility of $\mathrm{TgF}$ RT has been established as the standard of care. The issue that remains outstanding relates to the benefit to include the lymphatics.

Our study was undertaken to determine the dose distribution in the sentinel lymph node biopsy area (SLNBa) marked intraoperatively by clips. This could be helpful for RT optimization when only $\mathrm{TgF}$ are used for WBRT in patients with SLN involvement without ALND completion.

\section{METHODS}

This prospective study included 25 patients who have undergone BCS in a single institution between April 2012 and March 2013. The Henri Mondor Breast Center Multidisciplinary Committee has approved the protocol. Patients' characteristics are presented in Table 1.

\section{Surgery Procedure}

All patients underwent BCS and SLNB procedure for invasive $\mathrm{BC}$ (tumor size $<3 \mathrm{~cm}, \mathrm{cN} 0$ ). SLNB mapping was performed using technetium-99 m-labelled human albumin colloid particles. Blue dye was not systematically administered. SLNs were identified with a gamma detecting probe and/or blue dyed. After SLNs removal two titanium clips were placed to mark the location. No ALND was performed in this study.

\section{Radiation Therapy Technique}

All patients had 3D-conformal RT. Two radiation oncologists contoured axilla nodal volumes, SLNBa, and organs at risk using the RTOG contouring atlas. ${ }^{7}$ The WBRT technique and indications followed the French guidelines described elsewhere. ${ }^{8}$ Height of $\mathrm{TgF}$ was defined individually to target the breast volume. This study evaluated SLNBa coverage by $\mathrm{TgF}$ as determined intraoperatively by clips placement. For height TgF analyses, $\mathrm{STgF}$ was defined with the superior border set at $2 \mathrm{~cm}$ below the humeral head, whereas HTgF consisted of a superior border placed at the inferior edge of the humeral head.

The SLNBa was defined as a clinical target volume $\left(\mathrm{CTV}_{\mathrm{SLNB}}\right)$ with $5 \mathrm{~mm}$ in diameter surrounding the clips. To account for position uncertainties, we defined SLNBa planning target volume $\left(\mathrm{PTV}_{\mathrm{SLNB}}\right)$ with a $10 \mathrm{~mm}$ extension around the $\mathrm{CTV}_{\mathrm{SLNB}}$ (Fig. 1). Only two patients had seroma in SLNBa with a maximum diameter of 24 and $10 \mathrm{~mm}$. In both cases, clips were not displaced by the seroma cavity. The latter was included in the PTV $\mathrm{PLNB}_{\mathrm{SLN}}$ (Fig. 1).

TABLE 1 Patients' characteristics

\begin{tabular}{|c|c|}
\hline Median age (range) (year) & $61(43-84)$ \\
\hline \multicolumn{2}{|l|}{ Clinical tumor classification } \\
\hline Tis & 1 \\
\hline T1a & 4 \\
\hline $\mathrm{T} 1 \mathrm{~b}$ & 8 \\
\hline T1c & 12 \\
\hline \multicolumn{2}{|l|}{ Pathologic nodal status } \\
\hline pNO & 23 \\
\hline pN1 (mi) & 1 \\
\hline pN1a & 1 \\
\hline \multicolumn{2}{|l|}{ Hormone receptor and Her 2 status } \\
\hline Hormone receptors + & 23 \\
\hline Hormone receptors - & 2 \\
\hline HER2 positive & 2 \\
\hline \multicolumn{2}{|l|}{ Tumor differentiation } \\
\hline Grade I & 9 \\
\hline Grade II & 15 \\
\hline \multicolumn{2}{|l|}{ Pathology } \\
\hline Ductal invasive carcinoma & 22 \\
\hline Lobular invasive carcinoma & 2 \\
\hline Carcinoma in situ & 1 \\
\hline \multicolumn{2}{|l|}{ RT parameter } \\
\hline \multicolumn{2}{|l|}{ Total dose (Gy) } \\
\hline With boost (60-66 Gy) & 21 \\
\hline Without boost (40-50 Gy) & 4 \\
\hline Median delay from BCS-RT (range) (day) & $43(13-50)$ \\
\hline \multicolumn{2}{|l|}{ Patient morphology } \\
\hline Mean weight (range) (kg) & $67(47-102)$ \\
\hline Mean size $(\mathrm{cm})$ & 164 \\
\hline $\operatorname{BSA}\left(\mathrm{m}^{2}\right)$ & $1.74(1.46-2.17)$ \\
\hline Tangential fields thickness $(\mathrm{cm})$ & $13.6(9-18)$ \\
\hline
\end{tabular}

$B C S$ breast conserving surgery; $R T$ radiotherapy; $B S A$ body surface area 
FIG. 1 Topographic distribution of the clip locations, volumes and isodoses (95 and $50 \%$ ). a Example of clips topography in the sentinel lymph node biopsy area without associated seroma and tangential fields including totally the PTV $\mathrm{SLNB}_{\text {. }} C T V_{S L N B}$ clinical target volume of the sentinel lymph node biopsy area (in red); $P T V_{S L N B}$ planning target volume of the sentinel lymph node area (in light blue). b Example of clips topography in the sentinel lymph node biopsy area with associated seroma and tangential fields including partially the PTV $_{\text {SLNB. }}$ TgF were not adjusted to include totally the SLNBa in patients with negative SLN status. $C T V_{S L N B}$ clinical target volume of the sentinel lymph node biopsy area (in red); $P T V_{S L N B}$ planning target volume of the sentinel lymph node area (in blue).

c Example of PTV $\mathrm{V}_{\mathrm{SLNB}}$ and axilla levels CTV coverage by 95 and $50 \%$ isodoses using standard tangential fields (a)
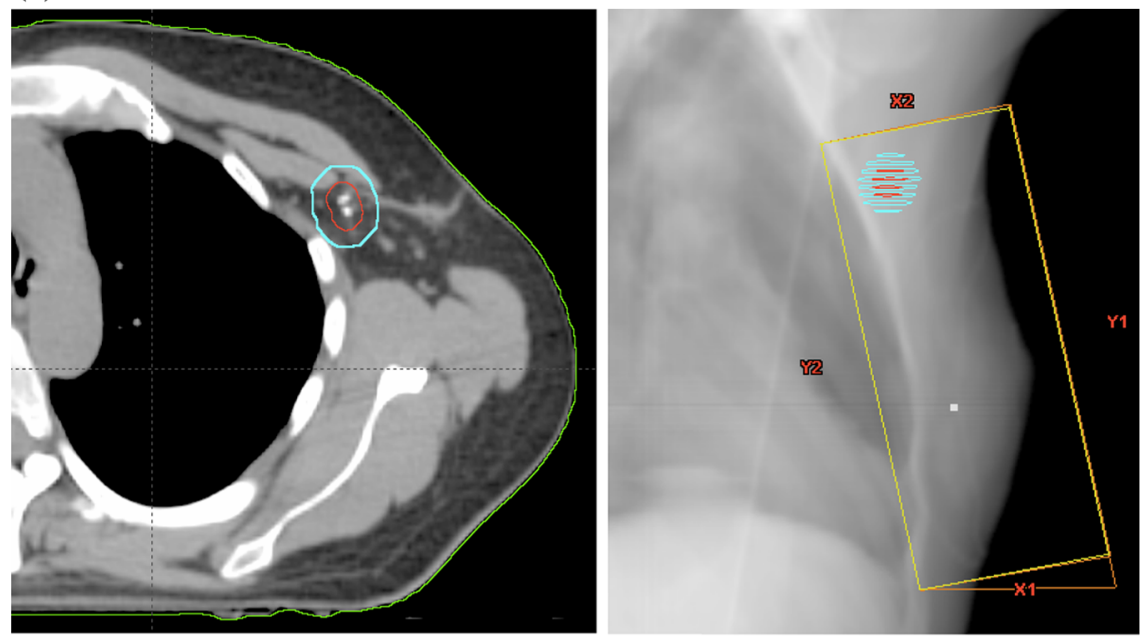

(b)
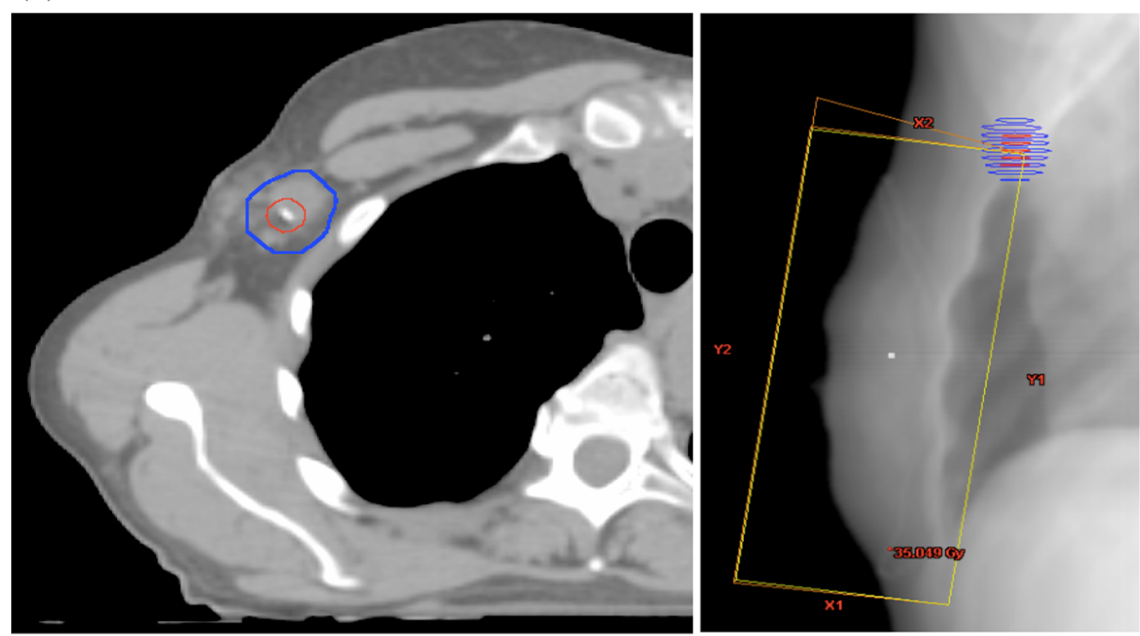

(c)

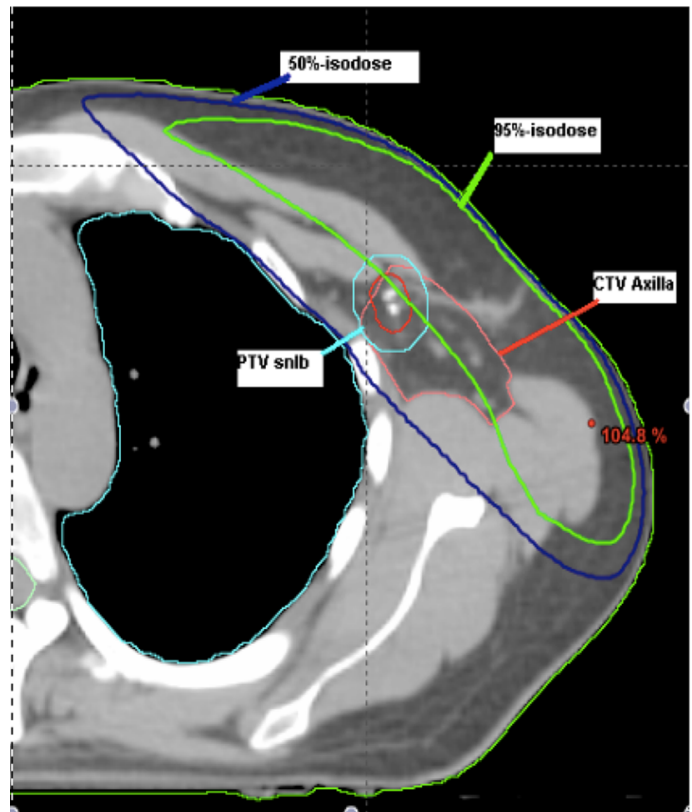




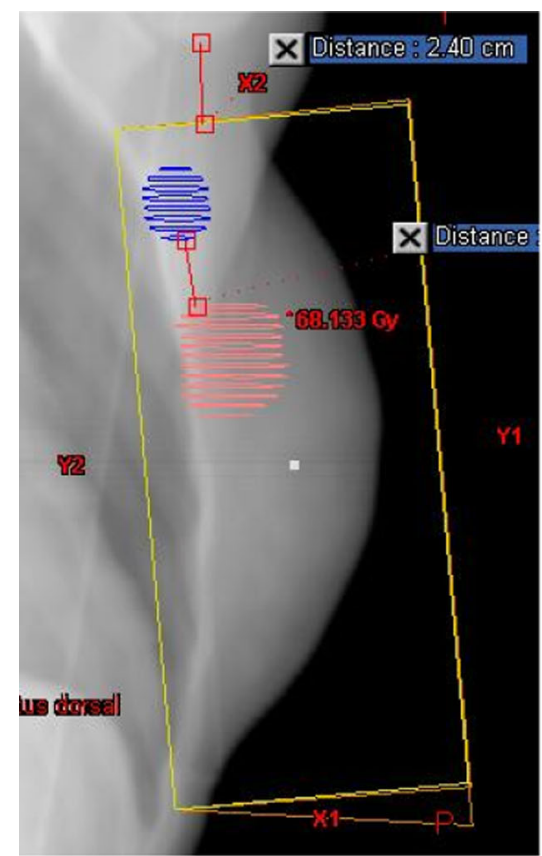

“Suitable group”(group I)

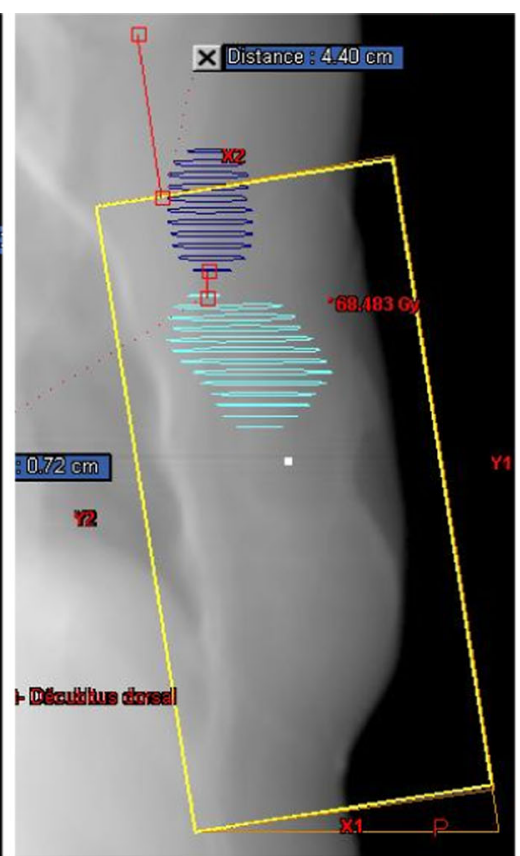

“Partially suitable”group (group II)

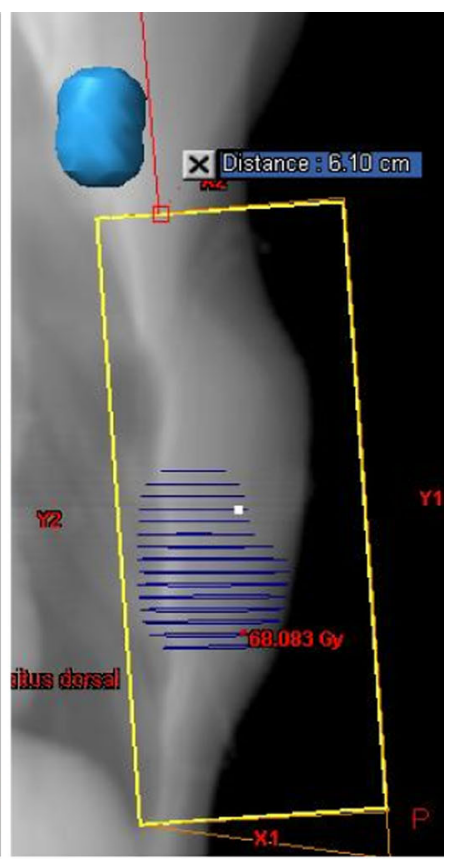

“Unsuitable"group (group III)
FIG. 2 Coverage of the sentinel lymph node biopsy area by tangential fields. Three groups of $\mathrm{TgF}^{\mathrm{PTTV}} \mathrm{V}_{\mathrm{SLNB}}$ overlap were defined: $100 \%$ overlap ("suitable group" with PTV SLNB $_{\text {completely }}$ included in the $\mathrm{TgF}$ ), $\geq 50 \%$ overlap ("partially suitable group" with

Dose-volume-histograms were analyzed according to axilla volumes receiving $95 \%$ (V95) or $50 \%$ (V50) of the prescribed dose. All values were compared according to the use of STgF $(n=20)$ or $\operatorname{HTgF}(n=5)$. Overlaps between the $\mathrm{TgF}$ and the $\mathrm{PTV}_{\mathrm{SLNB}}$ were analyzed in three groups of TgF-PTV $_{\text {SLNB }}$ overlap percentages: $100 \%$ overlap ("suit-

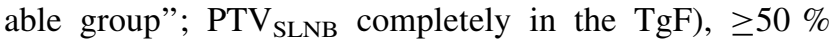
overlap ("partially suitable group"; PTV SLNB $_{\text {partially in }}$ the $\mathrm{TgF}$ ), and 0-49\% overlap ("unsuitable group"; $\leq 49 \%$ of the PTV $\mathrm{V}_{\mathrm{SLNB}}$ or completely outside the TgF; Fig. 2). Dose distribution was calculated in each of the three groups.

\section{Statistical Analyses}

All comparisons and correlations were performed using $t$ tests using SPSS software. Multiples comparisons were analyzed using an ANOVA post hoc Bonferroni. The level of significance was stated at $p<0.05$.

\section{RESULTS}

The median number of harvested SLNs was 1 (average 1.6; range 1-6). One patient had micrometastasis (MIC; $>0.2-2 \mathrm{~mm}$ ) and one had $1 \mathrm{MAC}$ out of 4 SLNs, respectively. Adjuvant systemic therapy and WBRT parameters are presented in Table 1.
PTV $_{\text {SLNB }}$ partially included in the TgF), and $0-49 \%$ overlap ("unsuitable group" with $50 \%$ of the PTV $\mathrm{PLNB}_{\mathrm{SLN}}$ or completely outside the TgF). Average dose was 46,34 , and $8 \mathrm{~Gy}$, respectively in the three groups

TABLE 2 Dose distribution in axilla levels I to III and the sentinel lymph node biopsy area

\begin{tabular}{|c|c|c|c|c|}
\hline RT parameters & Targets & Average (range) & & \\
\hline \multirow[t]{4}{*}{ Dose (Gy) } & Level I & $25(0-44)$ & & \\
\hline & Level II & $5(0-31)$ & & \\
\hline & Level III & $2(0-16)$ & & \\
\hline & $\mathrm{PTV}_{\text {SLNB }}$ & $33(1-60)$ & & \\
\hline \multirow[t]{4}{*}{ D95 (Gy) } & Level I & $5(0-36)$ & V95 (\%) & $2(0-23)$ \\
\hline & Level II & $1(0-3)$ & & 0 \\
\hline & Level III & $1(0-2)$ & & 0 \\
\hline & $\mathrm{PTV}_{\text {SLNB }}$ & $25(0-59)$ & & $4(0-99)$ \\
\hline \multirow[t]{4}{*}{ D50 (Gy) } & Level I & $30(1-49)$ & V50 (\%) & $47(0-96)$ \\
\hline & Level II & $4(0-48)$ & & $4(0-59)$ \\
\hline & Level III & $2(0-7)$ & & $1(0-21)$ \\
\hline & $\mathrm{PTV}_{\mathrm{SLNB}}$ & $33(1-60)$ & & $65(0-100)$ \\
\hline
\end{tabular}

$R T$ radiotherapy; $D 95$ dose delivered to $95 \%$ of the target; V95 volume of the target receiving $95 \%$ of the prescribed dose; $D 50$ dose delivered to $50 \%$ of the target; V50 volume of the target receiving $50 \%$ of the prescribed dose; PTV planning target volume; $P T V_{S L N B}$ PTV of the sentinel lymph node biopsy area

\section{Dose and Volume Coverage}

The average doses delivered to axillary levels I, II, III, and SLNBa were 25, 5, 2, and 33 Gy, respectively. 
Whereas coverage of these four volumes by the $95 \%$ isodose was limited (0-4\%), the $50 \%$ isodose covered 47 , 4,1 , and $65 \%$, respectively (Table 2 ). The average doses delivered to these four volumes were higher using $\mathrm{HTgF}$ than $\mathrm{STgF}$ (38 vs. $22 \mathrm{~Gy}, p=0.004 ; 11$ vs. $3 \mathrm{~Gy}$, $p=0.019 ; \quad 5$ vs. $2 \mathrm{~Gy}, p=0.003 ; 45$ vs. $30 \mathrm{~Gy}$, $p=0.02$ ), respectively (Table 3 ). While average D50 were

TABLE 3 Dose distribution comparison in levels I to III and the sentinel lymph node biopsy area according to tangential fields height

\begin{tabular}{lllll}
\hline RT parameters & Mean values & \multicolumn{2}{l}{} & \multirow{2}{*}{$p$ value } \\
\cline { 2 - 4 } & Axilla contents & STgF & HTgF & \\
\hline Average dose (Gy) & Level I & 22 & 38 & 0.004 \\
& Level II & 3 & 11 & 0.019 \\
& Level III & 2 & 5 & - \\
& PTV & 30 & 45 & 0.02 \\
& Level I & 5 & 6 & \\
D95 & Level II & 1 & 2 & NS \\
& Level III & 1 & 1 & \\
& PTV & 22 & 33 & \\
& Level I & 26 & 45 & $<0.001$ \\
& Level II & 2 & 12 & $<0.001$ \\
& Level III & 1 & 3 & - \\
& PTV & 1 & 65 & 0.001 \\
\hline
\end{tabular}

D95 dose delivered to $95 \%$ of the target; $D 50$ dose delivered to $50 \%$ of the target; PTV planning target volume; $P T V_{S L N B}$ PTV sentinel lymph node biopsy area; $S T g F$ standard tangential fields; $H T g F$ high tangential fields; $N S$ not significant higher in HTgF versus STgF patients, no difference was observed for D95. The results are presented in Table 3.

\section{SLNB Area Coverage}

In the STgF group $(n=20)$, the coverage of SLNBa by the $\mathrm{TgF}$ was "suitable" in eight cases (40\%), "partially suitable" in six cases (30\%), and "unsuitable" in six cases $(30 \%)$. In the HTgF group, four and one patients were considered "suitable" or "partially suitable," respectively. Finally, the SLNBa was completely covered by the TgF in 12 of 25 patients ( $48 \%$ ), independent of the TgF size. In the two patients with involved SLNs, STgF were modified as HTgF to include totally the SLNBa.

The average dose delivered to the PTV $\mathrm{PLNB}_{\text {SL }}$ was lower in the "unsuitable" ( $8 \mathrm{~Gy}$ ) versus "partially suitable" (34 Gy) versus the "suitable" group ( $46 \mathrm{~Gy} ; p=0.01$ ). The difference also was significant in terms of the average D95 $(p=0.017)$ and D50 $(p=0.028)$ delivered to the PTV $_{\text {SLNB }}$ (Table 4).

\section{Organs at Risk Analyses: Ipsilateral Lung and Heart}

The percentage of ipsilateral lung volumes receiving 5 Gy (V5), 10 Gy (V10), 20 Gy (V20), and the average dose were calculated. There was no statistical difference between $\mathrm{HTgF}$ versus STgF patients for: V20 (7 vs. $6 \%$; $p=0.33$ ), V10 (13 vs. $10 \% ; p=0.33$ ), and V5 (26 vs. $20 \% ; p=0.33)$. The mean ipsilateral lung dose using $\mathrm{HTgF}$ was not significantly greater than $\mathrm{STgF}$ (6 vs. $5 \mathrm{~Gy}$; $p=0.2$ ). In the left $\mathrm{BC}$ patients, the mean heart dose was higher with $\mathrm{HTgF}$ versus STgF (2.6 vs. $1.4 \mathrm{~Gy} ; p=0.02$ ).

TABLE 4 Dose distribution comparison in axilla contents of patients groups determined regarding the overlap between the sentinel lymph node biopsy area and tangential fields

\begin{tabular}{|c|c|c|c|c|c|}
\hline RT parameters & $\begin{array}{l}\text { Axilla contents } \\
\mathrm{n}\end{array}$ & $\begin{array}{l}\text { "Suitable" group (G I) } \\
12\end{array}$ & $\begin{array}{l}\text { "Partially suitable" group (G II) } \\
7\end{array}$ & $\begin{array}{l}\text { "Unsuitable" group (G III) } \\
6\end{array}$ & $p$ value \\
\hline \multirow[t]{4}{*}{ Average dose (Gy) } & Level I & 31 & 29 & 8 & NS \\
\hline & Level II & 8 & 2 & 1 & 0.027 \\
\hline & Level III & 4 & 1 & 1 & NS \\
\hline & $\mathrm{PTV}_{\text {SLNB }}$ & 46 & 34 & 8 & 0.01 \\
\hline \multirow[t]{4}{*}{ D95 } & Level I & 8 & 3 & 0 & 0.03 \\
\hline & Level II & 1 & 1 & 0 & NS \\
\hline & Level III & 1 & 1 & 0 & - \\
\hline & $\mathrm{PTV}_{\text {SLNB }}$ & 42 & 14 & 1 & 0.017 \\
\hline \multirow[t]{4}{*}{ D50 } & Level I & 42 & 32 & 3 & 0.045 \\
\hline & Level II & 7 & 2 & 1 & - \\
\hline & Level III & 2 & 2 & 1 & - \\
\hline & $\mathrm{PTV}_{\text {SLNB }}$ & 47 & 31 & 7 & 0.028 \\
\hline
\end{tabular}

D95 dose delivered to $95 \%$ of the target; $D 50$ dose delivered to $50 \%$ of the target; PTV planning target volume; PTV $S L N B$ PTV sentinel lymph node biopsy area; $N S$ not significant 


\section{DISCUSSION}

Our group showed recently that $\mathrm{STgF}$ planed for breast RT does not allow adequate coverage of the axilla. ${ }^{9}$ These findings are important to consider in the context of the international guidelines on ALND avoidance when systemic therapy and RT are expected to cure potential residual disease in the axilla. ${ }^{1-5}$ However, in the past decade, several studies have shown that STgF fails to adequately treat levels I-II. ${ }^{1,9-15}$ In this context, two major points on optimal regional RT technique to cover the axilla correctly should be considered. First, recent data from randomized trials and a meta-analysis have shown that nodal RT increases overall survival and particularly distant metastases free-survival. ${ }^{16-18}$ Second, RT to the axilla has been shown as safe and equivalent to ALND in patients with MIC in SLNB. ${ }^{19}$ These results highlight the importance of redefining adjuvant nodal RT in the SLNB era.

There is a direct relationship between prognosis and the number of involved LNs. In patients with positive SLN, the percentage of the SLN occupied by tumors and the number of SLNs removed are independently predictive of non-SLN involvement. In addition, the non-SLN involvement negatively influences survival. ${ }^{20}$ Reed et al. reported that none of 13 patients with ITCs who underwent an ALND had additional positive nodes compared with $27 \%$ of patients with MIC. At 5 years, distant recurrence rates in SLNnegative, isolated tumor cells, MIC, and MAC groups were $6,8,14$, and $21 \%$, respectively. The presence of MIC in the SLN was associated with a significantly shorter diseasefree interval than was SLN negativity $(p<0.02) .^{21}$

The RT objectives in case of axilla residual disease are to reduce the risk of locoregional recurrence and to prevent distant metastases from axillary sanctuary as hypothesized by Hellman: "RT is stopping metastases at their source." 22 These two objectives are highly linked together and to the axilla contents dose. Francissen et al. reviewed 16 studies describing patients with MAC disease in the SLN without ALND completion. ${ }^{23}$ After 43 months of follow-up, they observed only 24 axillary recurrences out of 3,268 (0.7 \%) among whom three received $\mathrm{RT} .{ }^{23}$ The axillary recurrence rate is even lower in the database study by $\mathrm{Yi}$ et al. with only $0.1 \%$ among the 1,473 patients with MAC in the SLN. ${ }^{24}$ Other smaller cohorts studies showed rates between 0 and $7.1 \%$. ${ }^{1}$ However, there is a lack of RT technique data to conclude on the relationship between axilla underdosage and local recurrence. ${ }^{2,5}$ In our study, STgF coverage of SNLBa was complete ("suitable" group) in only eight cases $(40 \%)$, whereas $\mathrm{HTgF}$ covered the SLNBa in four of five $(80 \%)$ patients. Indeed, the significant variations of the anatomical location of the SLNs do not allow coverage of the SLNBa with STgF, which does not include the LNs at highest risk of containing tumor.
Therefore, some authors suggested removing the superiorposterior corner multileaf collimators of the $\mathrm{TgF}$ to cover axilla levels. ${ }^{13,25,26}$ In our study, STgF have been modified as HTgF to include the whole SLNBa in both patients with metastases in the SLN.

Tumoricidal radiation dose also should be questioned. The evaluation of the delivered doses to axilla contents in the Z0011 study remains uncertain. ${ }^{5}$ The recent report from Jagsi et al. based on a centralized review of 228 (out of $605)$ patients provided only partial results on RT technique. Among the 185 (out of 228) patients with TgF-only treatment there was sufficient data to evaluate $\mathrm{TgF}$ height in $142(76.8 \%)$ patients. Because RT parameters and nodal volumes details were lacking, they could not evaluate the dose distribution in the axilla. However, they showed that direct nodal irradiation technique was mainly used in case of multiple nodal involvements for better coverage of the axilla compared with the HTgF technique. ${ }^{6}$

Several reports have highlighted that axillary nodal coverage depends on the upper $\mathrm{TgF}$ border. Studies using STgF showed that only approximately $50 \%$ of level I and $20-30 \%$ of level II nodes might receive $95 \%$ of the prescribed dose. ${ }^{10,26-28}$ However, several of these early studies used conventional simulation with surgical clips as anatomic landmarks to evaluate the dose distribution. For example, Reed et al. showed that STgF fail to treat the axillary level I-II anatomic volume adequately, with approximately $50 \%$ receiving a therapeutic dose. They concluded that surgical clips from ALND grossly underestimate the level I-II axilla nodal volume and should not be relied on for therapy planning. ${ }^{29}$

The use of HTgF in patients with MIC in the SLN is an important issue to consider when no ALND is indicated. In the Z0011 trial, HTgF were used in 50 and $52.6 \%$ of patients randomized to the ALND and SLNB arms, respectively. Of note, only $43(19 \%)$ patients received direct regional RT using $\geq 3$ fields. In this small group receiving a third field, there was a trend suggesting that treatment with posterior axillary boost field was more common in patients who had SLNB alone (12/21 vs. 6/22; $p=0.0066)$ and those receiving nodal $\mathrm{RT}$ had greater number of LNs involved $(p<0.001){ }^{6}$ Axilla coverage may be paramount to locoregional control and to decreasing the risk of metastatic dissemination from residual uncontrolled disease in the axilla. This is particularly important to consider regarding the recent data on overall and metastatic free-survival benefits from nodal RT in large clinical trials. ${ }^{16-18}$

Our study was undertaken to address the question of dose distribution and SLNBa coverage according to $\mathrm{TgF}$ size. We showed that the SLNBa was completely covered by the $\mathrm{TgF}$ independently from its size in only $48 \%$ of the patients. The average dose in the SLNBa was $33 \mathrm{~Gy}$. 
However, there was significantly higher delivered dose using HTgF. The average dose is considered as nontumoricidal in at least the partially suitable (34 Gy) and unsuitable ( 8 Gy) groups (Table 4 ). To overcome the underdosage of axilla, some authors have suggested tailoring $\mathrm{TgF}$ to targets. Kiel et al. recommended that the cranial field edge should be $1.2 \mathrm{~cm}$ below the humeral head and that $2.5 \mathrm{~cm}$ of the lung be included in the breast $\mathrm{TgF}$ to adequately cover the axilla. ${ }^{27}$ Schlembach et al. demonstrated that the LNs at greatest risk are $2 \mathrm{~cm}$ below the humeral head in $95 \%$ of cases. ${ }^{26}$ Reznik et al. reported an increase of 20-30\% of the average dose delivered to axilla levels when using HTgF. ${ }^{10}$ Considering the same borders, these values were lower in our earlier study in which STgF was used in the majority of patients. ${ }^{9}$

Many authors have attempted to define the anatomical borders by surgically marked axilla volumes and evaluated dose distribution at each level. Krasin et al. showed that only 1 of 25 patients received $50 \mathrm{~Gy}$ in the Level I of the axilla, and no patient had an adequate coverage of the Level II-III. ${ }^{11}$ Reed et al. showed a significant volume difference between the anatomical and the surgically marked axillary volumes in 18 of 50 patients undergoing ALND with more adequate coverage of the axilla in the latter. ${ }^{29}$ Another way to define marked axilla is to use a sentinel clip at the caudal border in the anatomically defined axilla. Using this procedure, Orecchia et al. showed that only 1 of 15 patients received $40 \mathrm{~Gy}$ in the axilla in a context of significant volume reduction. ${ }^{15}$

In a context of the ALND avoidance in selected patients, the debate on the axilla underdosage by $\operatorname{TgF}$ has to be addressed as the risk of non-SLNs involvement may depend on the anatomic location of SLN and its degree of involvement. ${ }^{30}$ The latter as determined by conventional histology has been described as a predictive factor for additional axillary involvement. ${ }^{21}$ Thus, as studies that have been undertaken to quantify intraoperatively the total tumor load in the positive SLNs showed that it is possible to predict additional non-SLN metastasis in the axilla with a high specificity, the authors suggested that this could be used to guide decisions for ALND completion. ${ }^{30}$ From the radiation oncology view, for an adequate coverage of the axilla, the use of direct fields could be considered rather than $\mathrm{TgF}$ in the patients with SLN involvement without further ALND. ${ }^{19}$

\section{CONCLUSIONS}

In patients undergoing BCS followed by WBRT, STgF provide a limited coverage of the axilla contents and deliver a nontumoricidal dose to potential axillary residual disease. The RT technique to deliver adequate dose to axilla and SLNBa has to be standardized with the use of $\mathrm{HTgF}$ or direct fields as described in AMAROS trial. ${ }^{19}$ This is true, insofar as we have: only limited follow-up in the SLNB trials without RT technique details, no clear tumoricidal dose level for residual disease, and uncertainties on the disease in the remained axilla non-SLN.

\section{CONFLICT OF INTEREST None.}

OPEN ACCESS This article is distributed under the terms of the Creative Commons Attribution License which permits any use, distribution, and reproduction in any medium, provided the original author(s) and the source are credited.

\section{REFERENCES}

1. Rao R, Euhus D, Mayo HG, Balch C. Axillary node interventions in breast cancer: a systematic review. JAMA. 2013;310:1385-94.

2. Krag DN, Anderson SJ, Julian TB, et al. Sentinel lymph-node resection compared with conventional axillary-lymph-node dissection in clinically node-negative patients with breast cancer: overall survival findings from the NSABP B-32 randomized phase 3 trial. Lancet Oncol. 2010;11:927-33.

3. Goldhirsch A, Winer EP, Coates AS, et al. Personalizing the treatment of women with early breast cancer: highlights of the St Gallen International Expert Consensus on the Primary Therapy of Early Breast Cancer 2013; Ann Oncol. 2013;24:2206-23.

4. Lyman GH, Temin S, Edge SB, et al. Sentinel lymph node biopsy for patients with early-stage breast cancer: American Society of Clinical Oncology clinical practice guideline update. $J$ Clin Oncol. 2014;32:1365-83.

5. Giuliano AE, McCall L, Beitsch P, et al. Locoregional recurrence after sentinel lymph node dissection with or without axillary dissection in patients with sentinel lymph node metastases: the American College of Surgeons Oncology Group Z0011 randomized trial. Ann Surg. 2010;252:426-32.

6. Jagsi R, Ballman K, Chadha M, et al. Radiation field design on the ACOSOG Z0011 trial. SABCS 2013. Abstract. P5-14-19. [http://sabcs.posterview.com/PDFs/P5-14-19.pdf].

7. White J, Tai A, Arthur D, et al. Variability of target and normal structure delineation for breast cancer radiotherapy: an RTOG Multi-Institutional and Multiobserver Study. Int J Radiat Oncol Biol Phys. 2009;73(3):944-51. [Breast Cancer Atlas for Radiation Therapy Planning: Consensus Definitions. http://www.rtog. org/CoreLab/ContouringAtlases/BreastCancerAtlas.aspx].

8. Belkacemi Y, Fourquet A, Cutuli B, et al. French Expert Review Board of Nice/Saint-Paul de Vence. Radiotherapy for invasive breast cancer: Guidelines for clinical practice from the French expert review board of Nice/Saint-Paul de Vence. Crit Rev Oncol Hematol. 2011;79:91-102.

9. Belkacemi Y, Allab-Pan Q, Bigorie V, et al. The standard tangential fields used for breast irradiation do not allow optimal coverage and dose distribution in axillary level I-II and the sentinel node area. Ann Oncol. 2013;24:2023-8.

10. Reznik J, Cicchetti MG, Degaspe B, Fitzgerald TJ. Analysis of axillary coverage during tangential radiation therapy to the breast. Int J Radiat Oncol Biol Phys. 2005;61:163-8.

11. Krasin M, McCall A, King S, Olson M, Emami B. Evaluation of a standard breast tangent technique: a dose-volume analysis of tangential irradiation using three-dimensional tools. Int J Radiat Oncol Biol Phys. 2000;47:327-33. 
12. Aristei C, Chionne F, Marsella AR, et al. Evaluation of level I and II axillary nodes included in the standard breast tangential fields and calculation of the administered dose: results of a prospective study. Int J Radiat Oncol Biol Phys. 2001;51:69-73.

13. Alço G, Igdem SI, Ercan T, et al. Coverage of axillary lymph nodes with high tangential fields in breast radiotherapy. $\mathrm{Br} J$ Radiol. 2010;83:1072-6.

14. Ohashi T, Takeda A, Shigematsu N, et al. Dose distribution analysis of axillary lymph nodes for three-dimensional conformal radiotherapy with a field-in-field technique for breast cancer. Int $J$ Radiat Oncol Biol Phys. 2009;73:80-7.

15. Orecchia R, Huscher A, Leonardi MC, et al. Irradiation with standard tangential breast fields in patients treated with conservative surgery and sentinel node biopsy: using a threedimensional tool to evaluate the first level coverage of the axillary nodes. Br J Radiol. 2005;78:51-4.

16. Whelan T, Olivotto I, Ackerman I, et al. NCIC-CTG MA 20: an intergroup trial of regional nod- al irradiation in early breast cancer. J Clin Oncol. 2011;29(Suppl): abstract LBA 1003.

17. Poortmans $\mathrm{P}$, Kirkove $\mathrm{C}$, Budach $\mathrm{V}$, et al. Irradiation of the internal mammary and medial supraclavicular lymph nodes in stage I to III breast cancer: 10 years results of the EORTC Radiation Oncology and Breast Cancer Groups phase III trial 22922/10925. EJC. 2013;47(Suppl 2).

18. Budach W, Kammers K, Boelke E, et al. Adjuvant radiotherapy of regional lymph nodes in breast cancer-a meta-analysis of randomized trials. Radiat Oncol. 2013;8:267.

19. Rutgers EJ, Donker M, Straver ME, et al. Radiotherapy or surgery of the axilla after a positive sentinel node in breast cancer patients: final analysis of the EORTC AMAROS trial (10981/ 22023). J Clin Oncol. 2013;31(Abstract LBA1001).

20. Pepels MJ, Vestjens JH, de Boer M, et al. Models predicting nonsentinel node involvement also predict for regional recurrence in breast cancer patients without axillary treatment. Eur J Surg Oncol. 2013;39:1351-7.

21. Reed J, Rosman M, Verbanac KM, et al. Prognostic implications of isolated tumor cells and micrometastases in sentinel nodes of patients with invasive breast cancer: 10 -year analysis of patients enrolled in the prospective East Carolina University/Anne Arundel Medical Center Sentinel Node Multicenter Study. J Am Coll Surg. 2009;208(3):333-40.

22. Hellman S. Stopping metastases at their source. $N$ Engl J Med. 1997;337:996-7.

23. Francissen TP, Dings Pim JM, Van Dalen T, Strobbe LJA, et al. Axillary recurrence after a tumor-positive sentinel lymph node biopsy without axillary treatment: a review of the literature. Ann Surg Oncol. 2012;19:4140-9.

24. Yi M, Giordano SH, Meric-Bernstam F, et al. Trends in and outcomes from sentinel lymph node biopsy (SLNB) alone vs. SLNB with axillary lymph node dissection for node-positive breast cancer patients: experience from the SEER database. Ann Surg Oncol. 2010;3:343-51.

25. Rabinovitch R, Ballonoff A, Newman F, Finlayson C. Evaluation of breast sentinel lymph node coverage by standard radiation therapy fields. Int J Radiat Oncol Biol Phys. 2008;70:1468-71.

26. Schlembach PJ, Buchholz TA, Ross MI, et al. Relationship of sentinel and axillary level I-II lymph nodes to tangential fields used in breast irradiation. Int $J$ Radiat Oncol Biol Phys. 2001;51:671-8.

27. Kiel KD CS, Small W. Is it possible to treat the axillary nodes in the same radiation fields covering the breast? A study to locate the limits of the axillary dissection relative to anatomic landmarks in the tangential fields. Int J Radiat Oncol Biol Phys. 1997;39:264.

28. Smitt MC, Goffinet DR. Utility of three-dimensional planning for axillary node coverage with breast-conserving radiation therapy: early experience. Radiology. 1999;210:221-6.

29. Reed DR, Lindsley SK, Mann GN, et al. Axillary lymph node dose with tangential breast irradiation. Int J Radiat Oncol Biol Phys. 2005;61:358-64.

30. Espinosa-Bravo M, Sansano I, Pérez-Hoyos S, et al. Prediction of non-sentinel lymph node metastasis in early breast cancer by assessing total tumoral load in the sentinel lymph node by molecular assay. Eur J Surg Oncol. 2013;39:766-73. 